\title{
Accidentes laborales durante la práctica clínica en estudiantes de la carrera de enfermería.
}

Título en inglés

\author{
Katiuska Susana Avila Fernández¹, Yaritza Lisbeth Cedillo Pacheco ${ }^{2}$, Janette \\ Esther Eras Carranza ${ }^{3}$ \\ $1-2-3$ Carrera de Enfermería. Unidad Académica de Ciencias Químicas y de la \\ Salud. Universidad Técnica de Machala
}

\section{Resumen}

Introducción: El rol de Enfermería, requiere tres elementos: conocimiento, actitudes, destreza, en este sentido, el riesgo que están expuestos los profesionales es desde el inicio de las prácticas en su formación académica; se conoce que los accidentes a los que están expuestos diariamente son: exposiciones fluidos, pinchazos, agresiones; por tal motivo se debe tomar en consideración la bioseguridad para disminuir debilidades por falta de experiencia, en pro de la salud y prevención de la enfermedad desde internado.

Objetivo: Analizar los accidentes laborales que se dan durante la práctica clínica en estudiantes de enfermería de la Universidad Técnica De Machala.

Métodos: Se basa en una investigación cuantitativa, observacional, analítico, transversal en la Carrera de Enfermería de la Universidad Técnica de Machala, durante el periodo de Octubre a Diciembre de 2017. Se realizó 166 estudiantes (78 octavo, 52 noveno, 36 décimo semestre). El método escogido es la encuesta, y el consentimiento informado.

Resultados: No existe asociación entre la variable de estudio de conocimientos de bioseguridad, con accidente laboral $\left(X^{2}=0,2287, p=0,63250021\right)$, la causa poca adherencia al usar protección, prisas en ejecutar tareas, observándose que $47 \%$ de estudiantes tuvo un accidente laboral, especialmente los que iniciaron su internado.

Conclusión: A pesar de que existe conocimiento en internos de Enfermería, se presentaron accidentes laborales durante las prácticas clínicas quedando evidenciado que la aplicación de la teoría con la práctica depende del estudiante y de la prioridad que le dé a su autocuidado.

Palabras clave: Accidentes laborales, estudiantes de enfermería, práctica clínica, bioseguridad, riesgos laborales. 


\section{Introducción}

El rol de Enfermería, requiere triangular tres elementos como son: conocimiento, actitudes y prácticas, en este sentido, el riesgo al que están expuestos los profesionales es desde el inicio debido a las prácticas que deben realizar desde su formación, los mismos que son considerados como accidentes de trabajo. Dentro de las definiciones de accidentes de trabajo surgen un sinnúmero de autores que presentan similares conceptualizaciones que giran sobre un mismo entorno; dentro de éstas se menciona a: todo incidente, inesperado, ocasionado en el trabajo de efecto rápido, que produce en el trabajador impedimentos temporales, permanentes o incluso la muerte. ${ }^{1}$, según la Organización Internacional del Trabajo (OIT), en promedio, 42 de cada 1000 trabajadores se accidentan cada año, en el mundo más de 313 millones de trabajadores sufren accidentes del trabajo y enfermedades profesionales no mortales, lo que equivale a 860000 víctimas al día. ${ }^{2}$ El riesgo de adquirir una enfermedad es uno de los peligros más frecuentes al que se encuentran expuestos los profesionales en sus ocupaciones. ${ }^{3}$

Por tal motivo la Organización Internacional del Trabajo (OIT) y la Organización Mundial de la Salud (OMS) son entidades que se preocupan por la salud y bienestar del trabajador, solicitando a los estados miembros elaboren programas de salud ocupacional y campañas de inmunización contra la hepatitis B $(37 \%$ de los trabajadores de la salud estuvieron expuestos a este virus, mientras que al de inmunodeficiencia adquirida menos del 10\% de trabajadores fueron expuestos), además señala que el $95 \%$ de las exposiciones a microorganismos transmitidos por vía sanguínea se puede evitar con medidas de protección seguras, y recibiendo las tres dosis necesarias de la vacuna de la hepatitis B. ${ }^{4}$

Así mismo la OMS señala que los riesgos laborales entorno al profesional de la salud se encuentran: los biológicos (microorganismos patógenos); químicos (sustancias tóxicas irritantes perjudiciales para la salud); físicos (como caídas); ergonómicos (relacionadas a mecánica corporal); psicosociales (agresiones físicas o verbales, factores estresantes). ${ }^{5}$ Dentro del sector de la salud, los riesgos potenciales más comunes, son los accidentes biológicos, Báez Pérez menciona que cada año, 3 millones de trabajadores están expuestos a contagios durante sus actividades diarias, dos millones al Virus de Hepatitis B, 0,9 millones a Hepatitis C y 170000 al Virus de Inmunodeficiencia Adquirida, siendo la Hepatitis B el de mayor incidencia. Por su parte los estudiantes de Enfermería, no están exentos a riesgos más aún ya que carecen de experiencia y habilidades suficientes, circunstancia que los hace más vulnerables, e indefensos y por tanto, más proclives a sufrir accidentes biológicos que pongan en deterioro su salud ${ }^{6}$ En este contexto Castro Fuentes señala que los accidentes con riesgo biológico en la salud, son una realidad a la que están expuestos todos los profesionales y concuerda que los agentes biológicos implicados destacan: Hepatitis B, C y de la Inmunodeficiencia Humana, por las graves consecuencias al trabajador. ${ }^{7}$

Puig Llobet señala que los estudiantes con aprendizaje práctico tienen mayor conocimiento del riesgo que se enfrenta diariamente el personal de enfermería, mientras que los estudiantes que no tienen formación práctica están más expuestos a sufrir cualquier daño que perjudique su estado de salud. ${ }^{8}$ Hernández en el 2012 señala que: hay un porcentaje alto de estudiantes que poseen conocimientos acerca de bioseguridad y que se dan varias falencias en las prácticas clínicas en el uso de 
barreras, eliminación de desechos y la realización de procedimientos antes y después de cada proceso. ${ }^{9}$

A propósito de lo antes mencionado Suelen Veras Gomes describe: Los estudiantes creen que los accidentes de trabajo están relacionados a los riesgos durante las actividades de práctica, reflejándose en su salud física y mental. El riesgo biológico por perforación cortante tuvo relevancia para la ocurrencia de accidente de trabajo. ${ }^{10}$, el conocimiento exacto de riesgo biológico en los estudiantes permitirá realizar intervenciones efectivas en función de la prevención en la práctica clínica de los estudiantes de la Carrera de Enfermería.

En relación a la inmunización, Wojciechowski et al, menciona que a pesar que los estudiantes consideran importante la inmunización como protección, el abordaje del tema en su formación es pequeño. Identifica que los estudiantes enfatizan la importancia de la prevención, sin embargo, muchos no tienen su calendario vacunal completo. ${ }^{11}$

Por otra parte con respecto a estadísticas, al referirse a estudiantes de enfermería, son pocos los estudios realizados sobre accidentes laborales, según datos encontrados de años anteriores, se pudo obtener que: en el 2001; se realizó un análisis en profesionales de enfermería donde 299 accidentes de trabajo fueron notificados; el accidente más frecuente correspondió a punción $(48,5 \%)$, seguido de traumatismos $(17,1 \%)$ y heridas cortantes $(12,4 \%) .{ }^{12}$

En el año 2003, Ortiz Molina mediante un estudio muestra que "el $42,2 \%$ de los alumnos tuvo algún accidente, debido a pinchazos (68,97\%) y salpicaduras $(57,47 \%)$. Las más frecuentes son dejar agujas u objetos cortantes en la batea $(82,5 \%)$, llevar materiales de riesgo en la mano $(62,1 \%)$ y reencapsular jeringuillas $(53,9 \%) "{ }^{13}$

Mientras que en el 2004, la incidencia de exposiciones con aguja canulada, es del $87 \%$ de 16.374 accidentes percutáneos de los cuales han sido 11,8 exposiciones por cada 100 camas. ${ }^{14}$

Entre los estudios realizados en estudiantes y profesionales de salud expuestos a material biológico contaminado Mendes de Almeida et al, señala que 461 personas se atendieron, siendo $389(84,4 \%)$ profesionales y $72(15,6 \%)$ estudiantes de la salud que sufrieron exposición a material biológico. ${ }^{15}$

Por otra parte López Siesto, expresa que los accidentes que se dan en el campo de la enfermería el $46,3 \%$ pertenecen a accidentes percutáneos, debido al poco conocimiento que los estudiantes tienen sobre bioseguridad. ${ }^{16} \mathrm{~A}$ propósito de los accidentes con materiales punzantes Jessyka Macedo, señala que a pesar de las precauciones estándar se recomienda el no reencapuche de agujas, no se adopte en las labores diarias de los profesionales de enfermería, propiciando mayor riesgo de accidentes ${ }^{17}$ enfatiza en la capacitación permanente y promueve a los profesionales a realizar sus prácticas diarias con responsabilidad.

Valverde Márquez, en su artículo; accidentes de trabajo y autoestima de profesionales de enfermería en ambientes hospitalarios; observó a través del análisis que: las causas como tabaquismo, religión, tiempo, pueden llevar al profesional al accidente y provocar cambios en el autoestima, pudiendo complicar su salud, calidad de vida y trabajo. ${ }^{18}$

Los accidentes con material biológico están vinculados al uso inadecuado y no adhesión de medidas de protección, sobrecarga de trabajo y autoconfianza. Resalta 
la importancia de elaborar políticas públicas en salud, buscando acrecentar las condiciones de trabajo y satisfacción profesional. ${ }^{19}$

Aline da Rocha, señala que: las medidas de bioseguridad, los programas educativos, la vacunación y la utilización de dispositivos deben ser implementados para evitar la exposición ocupacional. Por lo que enfermería se debe humanizar acerca de los peligros a los que están expuestos para proteger su salud. ${ }^{20}$ Tamayo Díaz demostró que mediante datos alcanzados las personas tienen conocimiento de los riesgos que están expuestos y que pueden causar los accidentes biológicos pero no emplean las medidas de protección estándar para evitar accidentes. ${ }^{21}$

La problemática planteada en los accidentes laborales durante la práctica clínica en estudiantes de la carrera de enfermería, se discierne que los accidentes a los que están expuestos diariamente en el cuidado directo al paciente son varios entre los que se encuentran; exposiciones directo a los fluidos con potenciales de riesgos, los pinchazos, las agresiones físicas o verbales entre otras; y la inexperiencia en la que nos toca enfrentar el día a día, hacen de la bioseguridad un arma eficaz para evitar estos inconvenientes.

Por tal motivo se deben asumir medidas de bioseguridad que permitan disminuir las debilidades en relación a la falta de experiencia en los estudiantes y enriquecer a través de la capacitación de ellos en el mejoramiento de estas prácticas hospitalarias, para lo cual se debe asumir acciones de promoción de la salud y prevención de la enfermedad; y dentro de la multiplicidad de funciones que tiene la enfermería, encontramos como esenciales la defensa y el fomento de un entorno seguro.

El objetivo de estudio es Analizar los accidentes laborales que se dan durante la práctica clínica, los conocimientos sobre bioseguridad y la correlación con las prácticas hospitalarias en los estudiantes de internado rotativo de la Carrera de Enfermería, mediante la aplicación de encuestas.

\section{Materiales y métodos}

Se realizó una investigación cuantitativa, con estudio observacional, analítico, transversal en la Carrera de Enfermería de la Unidad Académica de Ciencias Químicas y de la Salud de la Universidad Técnica de Machala, durante el periodo de Octubre a Diciembre de 2017. La muestra la configuraron tres grupos de estudiantes, de octavo semestre, que iniciaron su formación práctica (78 estudiantes), estudiantes de noveno semestre (52 estudiantes) que se encuentran culminado su formación práctica y aquellos estudiantes que aprobaron el internado rotativo y se encuentran cursando el décimo semestre (36 estudiantes); el criterio de inclusión fue: estar legalmente matriculados y aceptar participar en el estudio.

El método escogido es la encuesta, adaptada del instrumento de evaluación de riesgos laborales del Instituto de Seguros Sociales (ISS) ${ }^{9}$, la cual se aplicó con previa aprobación del estudiante y firma del consentimiento informado, en la cual se evalúa el nivel de conocimientos sobre bioseguridad y su correlación con las prácticas hospitalarias, el análisis estadístico se lo realizó a través del sistema computarizado Epi Info versión 3.5.2 para el cálculo del chi cuadrado y el valor de p..

\section{Resultados}


Tabla 1. Conocimientos de medidas de protección estándar relacionado con el accidente laboral en los estudiantes de internado rotativo de la carrera de enfermería. 2017

\begin{tabular}{cccc}
\hline $\begin{array}{c}\text { ¿Conoce las } \\
\text { medidas de } \\
\text { protección } \\
\text { estándar? }\end{array}$ & PRESENTO ACCIDENTE LABORAL & TOTAL \\
\hline Si & 86 & $\mathbf{S i}$ & \\
No & 2 & 77 & 163 \\
\hline Total & 88 & 1 & 3 \\
\hline
\end{tabular}

Fuente: Encuestas

Elaborado por: Equipo de investigación

Chi-square

Probability

0,2287

0,63250021

Análisis: el cuadro establece relación conocimientos de medidas de protección estándar relacionada con el accidente laboral en los estudiantes de internado rotativo de la carrera de enfermería. 2017

Se observa que el resultado del chi cuadrado es de 0,2287 , con el valor de $p$ de 0,63250021 , lo que indica que no existe asociación entre las variables de estudio, o sea que a pesar de que el conocimiento no es un causal para la presencia de un accidente laboral, y que independientemente del conocimiento que se tenga se presenta el accidente laboral e los internos de Enfermería en la práctica clínica.

Tabla 2. Frecuencia que ha sufrido accidente biológico relacionado con el sexo de los estudiantes de internado rotativo de la carrera de enfermería. 2017

\begin{tabular}{cccccc}
\hline Sexo & VECES & $\begin{array}{c}\text { QUE HA SUFRIDO UN ACCIDENTE } \\
\text { BIOLÓGICO }\end{array}$ & TOTAL \\
& Una & Dos & Tres o más & Ninguno & \\
\hline F & 34 & 21 & 11 & 73 & 139 \\
M & 7 & 5 & 0 & 15 & 27 \\
\hline Total & 41 & 26 & 11 & 88 & 166 \\
\hline
\end{tabular}

Fuente: Encuestas

Elaborado por: Equipo de investigación

Chi-square

Probability 
Análisis: el cuadro establece la relación entre las veces que ha sufrido un accidente biológico con el sexo de los estudiantes de internado rotativo de la carrera de Enfermería. 2017.

Se observa que el resultado del chi cuadrado es de 2,2517, con el valor de $p$ de 0,5218 , lo que indica que no existe asociación entre las variables de estudio, o sea que el sexo de los estudiantes no tiene relación causal con haber padecido de un accidente biológico durante el internado rotativo.

Tabla 3. Accidente biológico relacionado con el curso que estaban los estudiantes de internado rotativo de la carrera de enfermería. 2017

\begin{tabular}{ccccc}
\hline Accidente laboral & \multicolumn{3}{c}{$\begin{array}{c}\text { ¿QUÉ CURSO/S ESTUDIABA } \\
\text { CUANDO OCURRIÓ EL } \\
\text { ACCIDENTE? }\end{array}$} & TOTAL \\
& Octavo & Noveno & Decimo & \\
\hline No & 2 & 0 & 86 & 88 \\
Si, pinchazo con aguja \\
$\begin{array}{c}\text { hueca } \\
\text { Si, pinchazo con aguja } \\
\text { sólida }\end{array}$ & 7 & 4 & 0 & 11 \\
$\begin{array}{c}\text { Si, corte/herida } \\
\text { Si, salpicadura de } \\
\text { sangre/fluidos }\end{array}$ & 13 & 0 & 0 & 13 \\
$\mathrm{Si}$, pinchazo + corte & 24 & 6 & 0 & 30 \\
\hline Total & 2 & 4 & 0 & 21 \\
\hline
\end{tabular}

Fuente: Encuestas

Elaborado por: Equipo de investigación

Chi-square

Probability

171,1015

0

Análisis: el cuadro establece la relación entre el accidente biológico con el curso que estaban los estudiantes de internado rotativo de la carrera de enfermería. 2017. Se observa que el resultado del chi cuadrado es de 171,1015, con el valor de $p$ de 0 , lo que indica que existe asociación entre las variables de estudio, es decir que según muestra la evidencia 88 encuestados manifestaron no haber presentado un accidente laboral a lo largo de su internado rotativo, mientras que 63 encuestados manifiestan haber tenido al menos un accidente laboral en octavo semestre, al inicio del internado rotativo. 
Tabla 4. Conocimiento de protocolo relacionado con el accidente laboral en los estudiantes de internado rotativo de la carrera de enfermería. 2017

\begin{tabular}{|c|c|c|c|}
\hline \multirow[t]{2}{*}{ Conoce protocolo } & \multicolumn{2}{|c|}{ Accidente laboral } & \multirow[t]{2}{*}{ Tota } \\
\hline & $\mathrm{Si}$ & No & \\
\hline $\mathrm{Si}$ & 53 & 73 & 126 \\
\hline No & 25 & 15 & 40 \\
\hline Total & 78 & 88 & 166 \\
\hline
\end{tabular}

Fuente: Encuestas

Elaborado por: Equipo de investigación

Chi cuadrado: 5.09

p: 0.024

Análisis: el cuadro establece la relación entre el conocimiento de protocolo con el accidente laboral en los estudiantes de internado rotativo de la carrera de enfermería. 2017

Se observa que el resultado del chi cuadrado es de 5.09, con el valor de $p$ de 0.024 , lo que indica que existe asociación entre las variables de estudio con la validez que da el valor de $p$. evidenciándose que la mayoría de estudiantes conocen el protocolo frente a un accidente laboral, por otra parte se puede reflejar que una minoría de encuestados que sufrieron accidentes laborales no tenían conocimientos de protocolos.

Tabla 5. Accidente laboral y si ha necesitado tratamiento en los estudiantes de internado rotativo de la carrera de enfermería. 2017

Accidente laboral

Tratamiento

Total

\begin{tabular}{cccc} 
& Si & No & \\
\hline Si & 5 & 73 & 78 \\
No & 0 & 8 & 88 \\
\hline Total & 5 & 161 & 166 \\
\hline
\end{tabular}

Fuente: Encuestas

Elaborado por: Equipo de

investigación 
Análisis: el cuadro representa la relación entre las variables del accidente laboral y si ha necesitado tratamiento en los estudiantes de internado rotativo de la carrera de enfermería. 2017

Se observa que el valor del chi cuadrado es de 5.82, y el valor de p es de 0.015 , lo que indica que existe asociación entre las variables de estudio con la validez que da el valor de p. la evidencia establece que 88 estudiantes no han sufrido algún tipo de accidentes laborales, mientras que 78 si lo han sufrido de los cuales 5 han tenido que recibir tratamiento.

Tabla 6. Conocimiento de protocolo relacionado con esquema de vacunación en los estudiantes de internado rotativo de la carrera de enfermería. 2017

\begin{tabular}{cccc} 
Conoce protocolo & Esquema vacunación & Total \\
& $\mathrm{Si}$ & No & \\
\hline $\mathrm{Si}$ & 69 & 57 & 126 \\
$\mathrm{No}$ & 31 & 9 & 40 \\
\hline Total & 100 & 66 & 166 \\
\hline
\end{tabular}

Fuente: Encuestas

Elaborado por: Equipo de investigación
Chi cuadrado: 6.55
p: 0.01

Análisis: el cuadro representa el conocimiento de protocolo relacionado con esquema de vacunación en los estudiantes de internado rotativo de la carrera de enfermería. 2017

Se aprecia que el valor del chi cuadrado es de 6.55, y el valor de $p$ es de 0.01 , lo que indica que existe asociación entre las variables de estudio con la validez que da el valor de p. lo que indica que la mayoría de estudiantes conocen el protocolo y poseen su esquema de vacunación completo como medida de bioseguridad, por otra parte se puede mencionar que una minoría de estudiantes encuestados no conocen el protocolo y no poseen el esquema de vacunación completa como también manifiestan de no recordar si su esquema está al día por tal motivo están expuestos a sufrir rápidamente enfermedades. 
Tabla 7. Frecuencia que ha sufrido un accidente biológico relacionado con el tipo de accidente laboral en los estudiantes de internado rotativo de la carrera de enfermería. 2017

\section{TIPO DE ACCIDENTE ¿Cuántas veces ha sufrido un accidente TOTAL LABORAL biológico?}

\begin{tabular}{|c|c|c|c|c|c|}
\hline & Una & Dos & $\begin{array}{l}\text { Tres o } \\
\text { más }\end{array}$ & Ninguna & \\
\hline Sacando sangre & 2 & 0 & 0 & 0 & 2 \\
\hline $\begin{array}{c}\text { Canalizando una vía } \\
\text { venosa }\end{array}$ & 7 & 4 & 0 & 0 & 11 \\
\hline $\begin{array}{l}\text { Administrando una } \\
\text { inyección }\end{array}$ & 9 & 2 & 2 & 0 & 13 \\
\hline $\begin{array}{l}\text { Re-encapsulando la } \\
\text { aguja }\end{array}$ & 14 & 12 & 4 & 0 & 30 \\
\hline Curando una herida & 8 & 7 & 4 & 0 & 19 \\
\hline $\begin{array}{c}\text { Manipulando } \\
\text { desechos/basura }\end{array}$ & 1 & 2 & 0 & 0 & 3 \\
\hline $\begin{array}{l}\text { Introduciendo la aguja } \\
\text { u objeto cortante en el } \\
\text { contenedor especifico }\end{array}$ & 0 & 0 & 1 & 0 & 1 \\
\hline $\begin{array}{c}\text { Preparando o } \\
\text { recogiendo } \\
\text { instrumental quirúrgico }\end{array}$ & 0 & 1 & 0 & 0 & 1 \\
\hline Ninguno & 0 & 0 & 0 & 86 & 86 \\
\hline Total & 41 & 28 & 11 & 86 & 166 \\
\hline \multicolumn{6}{|c|}{$\begin{array}{l}\text { Fuente: Encuestas } \\
\text { Elaborado por: Equipo de investigación }\end{array}$} \\
\hline Chi-square & Probability & & & & \\
\hline 189,6414 & 0 & & & & \\
\hline
\end{tabular}

Análisis: el cuadro establece la relación entre las veces que ha sufrido un accidente biológico relacionado con el tipo de accidente laboral en los estudiantes de internado rotativo de la carrera de enfermería. 2017

Se observa que el resultado del chi cuadrado es de 189,6414, con el valor de $p$ de 0 , lo que indica que existe asociación entre las variables de estudio, evidenciándose 
que re - encapsulando la aguja tiene 30 encuestados que han tenido este tipo de accidente laboral, seguido de curando una herida con 19 encuestados, siendo estos dos los que más número de veces, han sufrido estos accidentes, 13 mencionaron que administrando una inyección mientras que 11 manifestaron que canalizando una vía venosa durante el internado rotativo. Siendo el manejo de las jeringuillas en cual procedimiento que se lo involucre, es el riesgo que presentan los internos de Enfermería en su práctica clínica.

Tabla 8. Conocimiento de las medidas de protección estándar relacionado con la edad en los estudiantes de internado rotativo de la carrera de enfermería. 2017

Edad
CONOCIMIENTOS DE MEDIDAS DE PROTECCIÓN

\begin{tabular}{cccc} 
& Si & No & \\
\hline DE 20 - 25 A & 125 & 2 & 127 \\
DE 26 - 31 A & 28 & 1 & 29 \\
DE 32 - 36 A & 8 & 0 & 8 \\
DE 37 A MAS & 2 & 0 & 2 \\
\hline Total & 163 & 3 & 166 \\
\hline
\end{tabular}

Fuente: Encuestas

Elaborado por: Equipo de investigación

\section{Chi-square Probability}

58,4194

0

Análisis: el cuadro establece la relación entre el conocimiento de las medidas de protección estándar con la edad en los estudiantes de internado rotativo de la carrera de enfermería. 2017

Se observa que el resultado del chi cuadrado es de 58,4194, con el valor de p de 0 , lo que indica que existe asociación entre la edad y el conocimiento de las medidas de protección estándar, ya que 125 internos de 20 a 25 años de edad si poseen conocimientos sobre el tema.

Tabla 9. Características de la ejecución de medidas de bioseguridad por parte de estudiantes de internado de enfermería. Universidad técnica de Machala 2017

\section{Frecuencia Realiza Lavado de}

\begin{tabular}{ccc} 
mano & Total & $\%$ \\
\hline Antes de cada procedimiento & 1 & 0,6 \\
Entre paciente a paciente & 2 & 1,2
\end{tabular}




\begin{tabular}{ccc} 
Después de cada procedimiento & 4 & 2,4 \\
En todas los casos anteriores & 159 & 95,8 \\
\hline Total & 166 & 100,0 \\
Hay que utilizar guantes & Total & $\%$ \\
\hline $\begin{array}{c}\text { Cuando prevea que puede } \\
\text { pincharse }\end{array}$ & 7 & 4,2 \\
$\begin{array}{c}\text { Cuando el paciente es de riesgo } \\
\text { Siempre que manipule material } \\
\text { biológico de pacientes }\end{array}$ & 5 & 3,0 \\
$\quad$ Ninguno & 120 & 72,3 \\
Total & 34 & 20,5 \\
\hline $\begin{array}{c}\text { Hay que utilizar batas, } \\
\text { mascarillas y protección ocular }\end{array}$ & 166 & 100,0 \\
\hline
\end{tabular}

Siempre que se pueda producir aerosoles o salpicaduras 92 55,4

Según el tipo de paciente 52 31,3

\begin{tabular}{ccc} 
Ninguno & 22 & 13,3 \\
\hline Total & 166 & 100,0
\end{tabular}

Conoce el protocolo que tiene que seguir en caso de accidente

\begin{tabular}{ccc} 
biológico & Total & $\%$ \\
\hline $\mathrm{Si}$ & 126 & 75,9 \\
$\mathrm{No}$ & 40 & 24,1 \\
\hline Total & 166 & 100,0
\end{tabular}

Posee usted el esquema de vacunación completo (hepatitis y difteria y tétano)

Total \%




\begin{tabular}{ccc} 
No & 100 & 60,2 \\
\hline Total & 166 & 100,0 \\
\hline
\end{tabular}

Fuente: Encuestas

Elaborado por: Equipo de investigación

Análisis: El cuadro establece las características de la ejecución de medidas de bioseguridad por parte de estudiantes de internado de Enfermería. Universidad Técnica de Machala 2017.

Se aprecia que entre las características de la ejecución de las medidas de bioseguridad, los estudiantes en la frecuencia de lavado de manos en el $95,8 \%$ lo realizan antes de cada procedimiento, entre paciente a paciente, y después de cada procedimiento; mientras que en la utilización de guantes, lo hacen siempre que manipule material biológico de pacientes en el $72,3 \%$; en cambio con el uso de batas, mascarillas y protección ocular, lo usan siempre que se pueda producir aerosoles o salpicaduras en el $55,4 \%$ de los estudiantes, en relación al conocimiento que tiene los estudiantes sobre protocolo que tiene que seguir en caso de accidente biológico, el 75,9\% lo conocen; mientras que en la administración del esquema de vacunación completo (hepatitis y difteria y tétano) que tiene el estudiante, el $60,2 \%$ no lo ha hecho.

Tabla 10. Características de la ejecución de medidas de bioseguridad específicas por parte de estudiantes de internado de enfermería. Universidad técnica de Machala 2017

Hay que encapsular las agujas antes de tirarlas

Total $\quad \%$

Sí, siempre que no tengamos el contenedor cerca 11 6,6

$\mathrm{Si}$, para evitar que la otra persona se pinche 60 36,1

\begin{tabular}{ccc} 
Nunca & 95 & 57,2 \\
\hline Total & 166 & 100,0
\end{tabular}

Sitio de depósito del material punzante o

\begin{tabular}{ccc} 
cortante & Total & $\%$ \\
\hline En el contenedor rígido específico & 154 & 92,8 \\
Dentro de su protector o capuchón & 7 & 4,2 \\
En la batea para trasladarlo de la habitación o & & \\
lugar donde se ha utilizado & 3 & 1,8 \\
Ninguno & 2 & 1,2 \\
\hline
\end{tabular}




\begin{tabular}{|c|c|c|}
\hline Total & 166 & 100,0 \\
\hline $\begin{array}{c}\text { Conocimiento de actividad que hacer } \\
\text { inmediatamente después de un pinchazo o } \\
\text { salpicadura? }\end{array}$ & Total & $\%$ \\
\hline Limpieza y desinfección & 132 & 79,5 \\
\hline $\begin{array}{l}\text { Lavado y desinfección solo cuando se trate de } \\
\text { un paciente de riesgo }\end{array}$ & 17 & 10,2 \\
\hline Ninguno & 17 & 10,2 \\
\hline Total & 166 & 100,0 \\
\hline $\begin{array}{l}\text { ¿Utiliza guantes para administrar tratamiento } \\
\text { intravenoso o canalizar vías? }\end{array}$ & Total & $\%$ \\
\hline Nunca & 11 & 6,6 \\
\hline Algunas veces & 65 & 39,2 \\
\hline Casi siempre & 37 & 22,3 \\
\hline Siempre & 53 & 31,9 \\
\hline Total & 166 & 100,0 \\
\hline $\begin{array}{c}\text { ¿Se ha puesto la bata, mascarilla y } \\
\text { protección ocular cuando ha habido riesgo } \\
\text { de salpicaduras? }\end{array}$ & Total & $\%$ \\
\hline Nunca & 12 & 7,2 \\
\hline Algunas veces & 44 & 26,5 \\
\hline Casi siempre & 47 & 28,3 \\
\hline Siempre & 63 & 38,0 \\
\hline Total & 166 & 100,0 \\
\hline
\end{tabular}

Fuente: Encuestas

Elaborado por: Equipo de investigación

Análisis: El cuadro establece las características de ejecución de medidas de bioseguridad específicas por parte de estudiantes de internado de enfermería. Universidad Técnica de Machala 2017 
Se aprecia que entre las características de la ejecución de las medidas de bioseguridad específicas, los estudiantes indican que hay que encapsular las agujas antes de tirarlas $51.2 \%$ estuvieron de acuerdo en que nunca se debe hacer; mientras que dónde hay que depositar el material punzante o cortante, 92,8\% manifestó que en el contenedor rígido específico; en cambio qué hay que hacer inmediatamente después de un pinchazo o salpicadura, el $79,5 \%$ realiza limpieza y desinfección, en relación al conocimiento que tiene los estudiantes sobre utilizar guantes para administrar tratamiento intravenoso o canalizar vías, se evidencia que $39,2 \%$, lo usan algunas veces; mientras que en, se ha puesto la bata, mascarilla y protección ocular cuando ha habido riesgo de salpicaduras, el $38 \%$ de los estudiantes manifestaron siempre.

Tabla 11. Características del accidente laboral por parte de estudiantes de internado de enfermería. Universidad técnica de Machala 2017

\begin{tabular}{ccc}
\hline ¿Cómo se produjo el accidente? & Total & $\%$ \\
\hline Sacando sangre & 6 & 3,6 \\
Canalizando una vía venosa & 17 & 10,2 \\
Administrando una inyección & 13 & 7,8 \\
Re-encapsulando la aguja & 9 & 5,4 \\
Curando una herida & 5 & 3,0 \\
Manipulando desechos - basura & 5 & 3,0 \\
Introduciendo la aguja u objeto cortante en el & 2 & 1,2 \\
contenedor especifico & 0 & 0,0 \\
Suturando & & \\
Preparando o recogiendo instrumental & 4 & 2,4 \\
quirúrgico & 19 & 11,4 \\
Otro & 86 & 51,8 \\
\hline Ninguno & 166 & 100,0 \\
\hline Total & Total & $\%$ \\
\hline CQué parte del cuerpo ha sido afectada? & 70 & 42,1 \\
\hline Dedos de la mano & 3 & 1,8 \\
\hline
\end{tabular}




\begin{tabular}{|c|c|c|}
\hline Ojos & 3 & 1,8 \\
\hline Brazos & 2 & 1,2 \\
\hline Otra zona & 2 & 1,2 \\
\hline Ninguno & 86 & 51,8 \\
\hline Total & 166 & 100,0 \\
\hline Frecuencia de accidente biológico & Total & $\%$ \\
\hline Una & 41 & 24,7 \\
\hline Dos & 28 & 16,9 \\
\hline Tres o más & 11 & 6,6 \\
\hline Ninguno & 86 & 51,8 \\
\hline Total & 166 & 100,0 \\
\hline $\begin{array}{c}\text { Aplicación de medidas de protección } \\
\text { momento del accidente }\end{array}$ & Total & $\%$ \\
\hline Si & 33 & 19,9 \\
\hline No & 47 & 28,3 \\
\hline Ninguno & 86 & 51,8 \\
\hline Total & 166 & 100,0 \\
\hline \multicolumn{3}{|l|}{ Percepción de la causa de accidente } \\
\hline biológico? & Total & $\%$ \\
\hline Falta de conocimiento & 4 & 2,4 \\
\hline Falta de habilidad & 16 & 9,6 \\
\hline Falta de precaución & 19 & 11,4 \\
\hline Prisas por exceso de tareas & 39 & 23,5 \\
\hline Otra & 2 & 1,2 \\
\hline Ninguno & 86 & 51,8 \\
\hline Total & 166 & 100,0 \\
\hline
\end{tabular}




\section{Fuente: Encuestas}

\section{Elaborado por: Equipo de investigación}

Análisis: El cuadro establece las características del accidente laboral por parte de estudiantes de internado de enfermería. Universidad técnica de Machala 2017

Se aprecia que entre las características del accidente laboral, los estudiantes en cómo se produjo el accidente el $10.2 \%$ manifiesta canalizando una vía venosa, y en qué parte del cuerpo ha sido afectada, $41.6 \%$ indica dedos de la mano; mientras cuántas veces ha sufrido un accidente biológico $24.7 \%$ manifestó una vez, en cambio cuando se produjo el accidente biológico, tenía la protección adecuada, el $28.7 \%$ no había aplicado medidas de protección; en relación a cuál cree que ha sido la causa de que haya tenido ese accidente biológico, $23.5 \%$ manifestó prisas por exceso de tareas.

\section{Discusión}

La hipótesis planteada de trabajo $\mathrm{H}_{1}$ Hay significancia estadística en la relación entre el conocimiento sobre bioseguridad y las prácticas clínica hospitalarias en los estudiantes de internado rotativo de la Carrera de Enfermería.

En cuanto a la relación entre los conocimientos de medidas de protección estándar relacionada con el accidente laboral el resultado del chi cuadrado es de 0,2287, con el valor de $p$ de 0,63250021 , lo que indica que no existe asociación entre las variables de estudio, o sea que independientemente del conocimiento que se tenga se presenta el accidente en la práctica clínica en los internos de Enfermería en la práctica clínica, y la magnitud de los accidentes en la práctica clínica presentó en el $47 \%$ de los estudiantes, al observar los resultados del presente estudio con el de Ortíz Molina señala que el $42,2 \%$ de los alumnos tuvieron algún tipo de accidente ${ }^{13}$, mientras que Hernández et al, indica en los resultados de su estudio que hay un porcentaje alto de conocimiento en los estudiantes acerca de bioseguridad, sin embargo que en las prácticas clínicas existen varias falencias en cuanto al uso de barreras de bioseguridad, la eliminación de desechos y la realización de procedimientos adecuados. ${ }^{9}$, otra situación a tomar en consideración es la frecuencia de los accidentes laborales que han sufrido relacionada con el sexo, en que el resultado del chi cuadrado fue de 2,2517, mientras que el valor de $p$ fue de 0,5218 , estableciéndose que no existe relación que independientemente del sexo se ha presentado el accidente biológico, ocurrido en 78 internos al menos una vez durante su práctica.

En relación al tipo de accidente biológico presentado relacionado con el semestre que cursaba, el resultado de chi cuadrado fue de 171,1015 y el valor de $p$ de 0 , lo que indica una fuerte asociación entre las dos variables de estudio, reflejándose que en los estudiantes de octavo semestre es donde más se presentaron los accidentes laborales, en el estudio de Moreno Arroyo et al, menciona que los estudiantes sin formación práctica previa tenían menor percepción de daño derivado del riesgo biológico con respecto a los estudiantes con formación práctica previa. ${ }^{24}$ se evidencia en los resultados del presente estudio que los estudiantes que mayormente presentaron accidente en la práctica clínica fueron los de octavo semestre, coincidiendo con el estudio de Moreno, de hecho los estudiantes de octavo semestre al iniciar su práctica clínica todavía no perciben el riesgo de la exposición a la ejecución de los procedimientos hospitalarios a pesar que 163 estudiantes si conocían acerca de las medidas de bioseguridad, el establecimiento 
de la conexión entre la teoría con la práctica depende mayormente de la prioridad que el estudiante da para hacer de este conocimiento una práctica en función de la prevención de los accidentes en la práctica clínica del internado rotativo.

En cuanto a la relación entre el conocimiento de protocolo con el accidente laboral en los estudiantes de internado rotativo de la carrera de enfermería. Se observa que el resultado del chi cuadrado es de 5.09, con el valor de $p$ de 0.024 , lo que indica que existe asociación entre las variables evidenciándose que la mayoría de estudiantes conocen el protocolo frente a un accidente laboral, por otra parte se puede reflejar que una minoría de encuestados que sufrieron accidentes laborales no tenían conocimientos de protocolos. Entre los datos obtenidos sobre conocimiento y conducta de bioseguridad Morais, reveló que el $51,4 \%$ conocía el protocolo; $22,9 \%$ tenían accidentes con material biológico; sólo el $14,3 \%$ notifico lavado con agua y jabón en lesión ante la ocurrencia de un accidente. La cual se demostró que hay falencias en relación con las acciones de bioseguridad ${ }^{23}$, en este contexto sobre la relación entre accidente laboral y el tratamiento en los estudiantes de internado rotativo de la carrera de enfermería 2017 se observa que el chi cuadrado es de 5.82, y el valor de $\mathrm{p}$ es de 0.015 , lo que indica que hay validez, la evidencia establece que 88 estudiantes no han sufrido algún tipo de accidentes laborales, mientras que 78 si lo han sufrido de los cuales 5 han tenido que recibir tratamiento.

En cuanto al conocimiento de protocolo y esquema de vacunación se aprecia que el valor del chi cuadrado es de 6.55 , y el valor de $p$ es de 0.01 , lo que indica que existe asociación entre las variables de estudio con la validez que da el valor de p. además demuestra que el $60 \%$ de estudiantes conocen el protocolo y poseen su esquema de vacunación completo como medida de bioseguridad, por otra parte se puede mencionar que $5.4 \%$ de estudiantes encuestados no conocen el protocolo y no poseen el esquema de vacunación completa por tal motivo están expuestos a enfermedades. Por su parte Wojciechowski et al., en su estudio cualitativo sobre la importancia de la inmunización los resultados revelaron que a pesar de que los estudiantes consideraron importante la inmunización como un modo de protección, el abordaje del tema en su formación todavía es muy pequeño. Identifica también que los estudiantes reconocen las inmunizaciones como modos de protección y enfatizan la importancia de la prevención, sin embargo, muchos no tienen su calendario vacunal completo ${ }^{11}$. En este entorno Arrieta menciona que la vacunación contra la hepatitis $B$ es un medio eficaz para disminuir el riesgo de adquisición del virus de la hepatitis $B(\mathrm{VHB})$ durante las prácticas de los alumnos. En ese estudio se encontró que $171(81,4 \%)$ sujetos tenían experiencia acerca de la vacunación para VHB, valores similares a los encontrados en Sudáfrica, quienes hallan que los estudiantes no tienen vacunación contra VHB. ${ }^{25}$ la de la vacunación es importante para los estudiantes de internado rotativo ya que se protegen contra una enfermedad como es hepatitis $B$, por el riesgo que emite en la transmisión de una persona a otra a través del manejo de material corto punzante en el manejo de las jeringuillas, bisturí entre otros.

En lo relativo a la frecuencia de accidentes biológicos con el tipo de accidente laboral en los estudiantes de internado rotativo de la carrera de enfermería, se observa que el resultado del chi cuadrado es de 189,6414, con el valor de p de 0 , lo que indica que existe una fuerte asociación entre las variables de estudio, estando 
reencapsulado la aguja $(38,4 \%)$, seguido de curando una herida $(25 \%)$, siendo estos dos los que más veces, han sufrido accidentes, 13 (17\%) mencionaron que administrando una inyección mientras que $11(6,6 \%)$ canalizando una vía venosa. Reflejándose que el manejo de las jeringuillas en el procedimiento que se lo involucre, es el mayor riesgo que presentan los internos de Enfermería en su práctica clínica. En concordancia a estos datos, Ortiz Molina refiere que su principal riesgo son los pinchazos $(68,97 \%)$ y salpicaduras $(57,47 \%)$. Las prácticas de riesgo más frecuentes son dejar agujas u objetos cortantes en la batea $(82,5 \%)$, llevar materiales de riesgo en la mano $(62,1 \%)$ y reencapsular jeringuillas $(53,9 \%) .{ }^{13}$

En lo referente al conocimiento de las medidas de protección estándar con la edad se visualiza que el resultado del chi cuadrado es de 58,4194, con el valor de $p$ de 0 , lo que señala que existe asociación entre la edad y el conocimiento, ya que 125 internos de 20 a 25 años de edad si poseen conocimientos sobre el tema. Por otra parte Velásquez Ormaeche, menciona sobre lo niveles de conocimientos en bioseguridad que el $53.2 \%$ fueron hombres y de todos solo 9 refirieron haber sufrido algún accidente intrahospitalario, y la edad promedio global fue de 23,50 años. ${ }^{22}$

Se aprecia que entre las características de la ejecución de las medidas de bioseguridad, en la frecuencia de lavado de manos el 95,8\% lo realizan antes de cada procedimiento, entre paciente a paciente, y después de cada procedimiento; mientras que en la utilización de guantes, lo hacen siempre que manipule material biológico, el $72,3 \%$; el uso de batas, mascarillas y protección ocular, lo usan siempre que se pueda producir aerosoles o salpicaduras en el $55,4 \%$ de los estudiantes, en relación al conocimiento que tiene los estudiantes sobre protocolo, el 75,9\% lo conocen; Arrieta et al. Menciona que el uso de barreras de protección personal que menos usan durante su práctica clínica, $204(97,7 \%)$ manifestaron que fueron las gafas, aun cuando 148 de ellos $(70,4 \%)$ conocía la existencia de riesgo de infección a través de las salpicaduras; situación que es similar a la notificada en otros estudios donde las gafas y la careta poseen los porcentajes más bajos de uso comparados con la utilización de otros elementos como guantes de látex y bata. ${ }^{25}$

Concerniente a las características de ejecución de medidas de bioseguridad específicas por parte de estudiantes de internado de enfermería. Indicaron que hay que encapsular las agujas antes de tirarlas $51.2 \%$ mencionaron en estar de acuerdo en que nunca se debe hacer; de acuerdo al estudio de Bautista Rodríguez el manejo de material punzo cortante es a menudo, debido a que el $74,29 \%$ del personal de enfermería práctica el reencapuchado de agujas con una sola mano. ${ }^{3}$ Mientras que dónde hay que depositar el material punzante, 92,8\% manifestó en el contenedor rígido específico; en este contexto los datos obtenidos en el estudio de Bautista Rodríguez et al, en cuanto al manejo de material corto punzante se evidencia un nivel de aplicación deficiente con un $64 \%$, regular con un $27 \%$ y un nivel bueno con $9 \%$. ${ }^{3}$ Lo cual difiere con el resultado obtenido ya que se observa un alto nivel de conocimiento en los estudiantes sobre donde depositar el material punzante.

Otras de las interrogantes es el qué hay que hacer inmediatamente después de un pinchazo o salpicadura, el 79,5\% realiza limpieza y desinfección, en relación al conocimiento que tiene los estudiantes sobre utilizar guantes para administrar 
tratamiento intravenoso o canalizar vías, se evidencia que $39,2 \%$, lo usan algunas veces; en el uso de guantes, un $45 \%$ del personal estudiado, considerando que solo este porcentaje presenta un nivel de conocimiento bueno. ${ }^{3}$

Mientras que en, se ha puesto la bata, mascarilla y protección ocular cuando ha habido riesgo de salpicaduras, el $38 \%$ de los estudiantes manifestaron siempre. Por otra parte el uso de gorro, bata y mascarilla actúan como barrera, respecto a ello el personal de Enfermería de la Clínica San José, presenta un conocimiento bueno en un $28 \%$, predominando en ellas el conocimiento regular equivalente a un $59 \%$. $^{3}$

Entre las características del accidente laboral por parte de estudiantes de internado de enfermería. Se observa que el $10.2 \%$ manifiesta canalizando una vía venosa, y en qué parte del cuerpo ha sido afectada, $41.6 \%$ indica dedos de la mano; mientras cuántas veces ha sufrido un accidente biológico $24.7 \%$ manifestó una vez, en cambio cuando se produjo el accidente biológico, tenía la protección adecuada, el $28.7 \%$ no había aplicado medidas de protección; en relación a cuál cree que ha sido la causa a las que se le atribuye es la poca adherencia que se tiene al usar la protección adecuada, observándose que el $28.7 \%$ no había aplicado medidas de protección; atribuyéndole a prisas por exceso de tareas. Mientras que en su estudio Hernández et al., menciona que "la incidencia de exposiciones con aguja canulada, es del $87 \%$ de 16.374 accidentes percutáneos de los cuales han sido 11,8 exposiciones por cada 100 camas. $^{14}$

\section{Conclusion}

Se evidencia que existen conocimientos sobre bioseguridad en los estudiantes de internado rotativo de la Carrera de Enfermería como fortaleza, no obstante se pudo reflejar que a pesar de que existe el conocimiento se presentaron accidentes laborales durante sus prácticas clínicas, hospitalarias en su mayor parte en estudiantes, que iniciaron la prácticas clínicas, en concordancia queda demostrado que el vínculo que existe entre la teoría con la práctica depende del estudiante y de la prioridad que le dé a la prevención de los accidentes en la práctica clínica del internado rotativo.

Se detectó además que los tipos de accidentes laborales que se dan con mayor frecuencia durante la práctica clínica son los pinchazos ya sea reencapsulando la aguja, administrando una inyección, canalizando vía venosa, o las salpicaduras de fluidos en procesos como curando una herida. El manejo de las jeringuillas en el procedimiento que mayor riesgo que presentan los internos de Enfermería en su práctica clínica. En consideración a estas situaciones de exposición a las cuales se enfrentan diariamente los estudiantes y en vista de que gran parte de ellos no posee el esquema de vacunación completo, se recomienda poner mayor énfasis en que los estudiantes se apliquen estas vacunas antes de ingresar a realizar su internado rotativo, con la finalidad de tener una protección adecuada al ingresar a realizar sus prácticas hospitalarias.

En relación a las características individuales de los estudiantes de enfermería, se pudo apreciar que ni la edad, ni el sexo son claves para que se presenten accidentes laborales, atribuyéndose más bien a lo que manifiestan los internos acerca de las prisas por exceso de tareas, a esto puede añadirse que la falta de destreza al realizar procedimientos por la poca experiencia previa son un factor determinante para que se presente un accidente laboral. 


\section{Referencias Bibliográficas}

1. Rodríguez Eduardo, Brunstein Luisa, Digón Ana. Glosario Temático de la Salud del Trabajador en el MERCOSUR, Ministerio de Salud de la Nacion. Programa de Salud del trabajador. [página en la Internet]. 2012 Jul [citado 2017 Sept 24]; 12. Disponible en: http://www.msal.gob.ar/images/stories/bes/graficos/0000000340cnt-11-Glosario salud tr abajador.pdf

2. OIT. Organización Internacional de Trabajo [página en la Internet]. 2017. [citado 2017 Sept 29]. Disponible en: http://www.ilo.org/global/topics/safety-and-health-at-work/lang--es/index.htm

3. Bautista-Rodríguez, Luz, Delgado-Madrid, Carmen, Hernández-Zárate, Zulma, Sanguino-Jaramillo, Fanny, Cuevas-Santamaría, Martha, Arias-Contreras, Yessenia, Mojica-Torres, Isabel. Nivel de conocimiento y aplicación de las medidas de bioseguridad del personal de enfermeria. Ciencia y Cuidado. [revista en la Internet] 2013 Dic. [citado 2017 Dic 08]; 10 (2): 128-133. Disponible en: https://dialnet.unirioja.es/descarga/articulo/4698254.pdf

4. OMS. Salud ocupacional del trabajador. World Health Organization [página en la Internet]. 2017. [citado 2017 Sept 29]. Disponible en: http://www.who.int/occupational health/topics/hcworkers/en/

5. Jiménez-Paneque, Rosa, Pavés-Carvajal, Juan. Enfermedades y riesgos laborales en trabajadores de servicios de urgencia: revisión de la literatura y acercamiento a Chile. Medwave. [revista en la Internet] 2015 Ago. [citado 2017 Oct 04]. Disponible en: http://www.medwave.cl/link.cgi/Medwave/Revisiones/RevisionTemas/6239.act?ver=sind iseno

6. Báez-Pérez, Carlos. Bioseguridad y accidentes biológicos en estudiantes de Enfermería. Facultad de Enfermeria e Podoloxia Universidad de Coruña [página en la Internet]. 2015 Dic. [citado 2017 Sept 24]; 7. Disponible en: http://ruc.udc.es/dspace/bitstream/handle/2183/16914/BaezPerez_Carlos_TFG_2015.pdf? sequence $=2 \&$ is Allowed $=y$

7. Castro-Fuentes, Leticia. El accidente con riesgo biológico en el sector sanitario. Repositorio Abierto de la Universidad de Cantabria UCrea [página en la Internet]. 2014 Abr. [citado 2017 Sept 24]. Disponible en: https://repositorio.unican.es/xmlui/handle/10902/5198?locale-attribute=fr

8. Puig-Llobet, Montserrat, Moreno-Arroyo, Ma. Carmen, Lluch-Canut, Ma. Teresa, Roldán-Merino, Juan, Casas-García, Irma, Falco-Pegueroles, Anna. ¿Qué percepción del riesgo biológico tienen los estudiantes de Grado de Enfermería? Dipòsit Digital de la Universitat de Barcelona [revista en la Internet] 2016 Jul. [citado 2017 Sept 24]. Disponible en: http://diposit.ub.edu/dspace/handle/2445/106568

9. Hernàndez, Anuar, Montoya, Jorge, Simancas, Miguel. Conocimientos, prácticas y actitudes sobre bioseguridad en estudiantes de odontología. Rev Colomb Investig Odontol 
[revista en la Internet] 2012 [citado 2017 Sept 27]; 9 (3). Disponible en: https://www.rcio.org/index.php/rcio/article/view/109

10. Veras-Gomes, Suelen, de-Araujo-Rodrigues, Clarice, Alves-Pereira, Érika, de-CastroHandem, Priscila, Pereira-Passos, Joanir. Accidentes en el trabajo en el campo de la práctica de los estudiantes de la educación. Revista Online de Pesquisa [revista en la Internet] 2015 [citado 2017 Sept 24]; 7 (4). Disponible en: http://www.seer.unirio.br/index.php/cuidadofundamental/article/view/4455

11. Wojciechowski-de-Carvalho, M., Zepka-Baumgarten, L., Riegert-Borba, M., Silva-Da-Costa, C. Percepción de los estudiantes de enfermería acerca de su protección ante patologías inmunoprevisibles. Revista Electronica Trimetral de Enfermeria [revista en la Internet] 2012 Ene [citado 2017 Sept 24]; (25). Disponible en: https://digitum.um.es/xmlui/bitstream/10201/27379/1/Percepcion $\% 20 \mathrm{de} \% 2010 \mathrm{~s} \% 20 \% 20 \mathrm{e}$ studiantes $\% 20 \mathrm{de} \% 20$ enfermeria $\% 20$ acerca $\% 20 \mathrm{de} \% 20$ su.pdf

12. Tomasina, Fernando, Gómez-Etchebarne, Fernando. Accidentes laborales en el Hospital de Clínicas. Rev Méd Uruguay [revista en la Internet]. 2001 Dic [citado 2017 Sept 27] ; (17): 156-160. Disponible en: http://www.rmu.org.uy/revista/2001v3/art2.pdf

13. Ortiz-Molina, Silvia. Riesgos biológicos de los estudiantes de enfermería. Enfermería Clínica: Elsevier [revista en la Internet]. 2003 [citado 2017 Sept 27]; 13 (5): 285-289. Disponible en: http://www.sciencedirect.com/science/article/pii/S1130862103738224

14. Hernández-Navarrete, María, Campins-Martí, Magda, Martínez-Sánchez, Elena, Ramos-Pérez, Francisca, García-de-Codes-Ilario, Aurelia, Arribas-Llorente, José, et al. Exposición ocupacional a sangre y material biológico en personal sanitario. Medicina Clinica: Elsevier [revista en la Internet]. 2004 [citado 2017 Sept 27]; 122 (3): 81-86. Disponible en: http://www.sciencedirect.com/science/article/pii/S0025775304741512

15. Mendes-de-Almeida, María, Marín-da-Silva,-Caninis, Reis, Renata, Malaguti-Toffano, Silmara, Vieira-Pereira, Fernanda, Gir, Elucir. Seguimiento clínico de profesionales y estudiantes del área de la salud expuestos a material biológico potencialmente contaminado. Revista da escola de enfermagem da USP. [revista en Internet] 2015 Abr. [citado 2017 Sep. 24]. Disponible en: http://www.journals.usp.br/reeusp/article/view/103195/101599

16. Siesto-López, Ángel. Accidente con riesgo biológico en los estudiantes de enfermería de la universidad de salamanca durante sus prácticas clínicas. Revista Enfermería CyL. [revista en Internet] 2017. [citado 2017 Sep. 24]. Disponible en: http://www.revistaenfermeriacyl.com/index.php/revistaenfermeriacyl/article/view/192/16 $\underline{7}$

17. Macedo-Mendes, Jessyka, Pereira-Rodrigues, Tiago, Fernandes-Morais, Lucineide. Riesgos biológicos y sus medidas preventivas en la práctica de los profesionales de enfermería en la atención básica. Revista Brasileña de Educación y Salud. [revista en 
Internet] 2014 Enero. [citado 2017 Sep. 24]. Disponible en: http://gvaa.com.br/revista/index.php/REBES/article/view/3159/2831

18. Valverde-Marques-dos Santos, Sergio, Ribeiro-Macedo, Flavia, Almeida da-Silva, Luiz, Rodrigues-Resck, Celia, Alves-Nogueira, Denismar, Fábio de, Souza Tierra. Accidente de trabajo y autoestima de profesionales de enfermería en ambientes hospitalarios. Revista Latinoamericana de Enfermería. [revista en Internet] 2017 Abr. [citado 2017 Sep. 24]. Disponible

en: http://www.scielo.br/scielo.php?pid=S0104-11692017000100328\&script=sci_arttext\&tln $\mathrm{g}=\mathrm{pt}$

19. Guedes dos Santos, José, Vieira Mariana, Ferreira-Cardoso, Luciana, Gomes, Doris, Schlindwein Betina, de-Azevedo Silvia. Riesgo y vulnerabilidad en las prácticas laborales de los profesionales del área de la salud. Revista Gaúcha de Enfermagem. [revista en Internet] 2012 Jun. [citado 2017 Sep. 24]. Disponible en: http://www.scielo.br/scielo.php?script=sci_arttext\&pid=S1983-14472012000200028

20. Rocha-Kallás, Aline da, Rueda-Almeida, Cristina. Accidentes ocupacionales con material biológico: la actuación del enfermero. Enfermagem Revista. [revista en Internet]. 2013 Sep. [citado 2017 Sep. 24]. Disponible en: http://periodicos.pucminas.br/index.php/enfermagemrevista/article/viewFile/12892/10115

21. Díaz-Tamayo, Alejandra, Vivas-M., Martha. Riesgo biológico y prácticas de bioseguridad en docencia. Facultad Nacional de Salud Pública. [revista en Internet]. 2016 Feb. [citado 2017 Dic 24]. 34 (1): 62-69. Disponible en: http://www.redalyc.org/html/120/12043924007

22. Velasquez-Ormaeche, Sonia, Factores asociados al nivel de conocimientos sobre bioseguridad en internos de medicina. Universidad Privada Antenor Orrego. [Tesis en internet] 1 Jun. [citado 2017 Sep. 24]. Disponible en: http://repositorio.upao.edu.pe/bitstream/upaorep/2739/1/RE_MED.HUMA_SONIA.VEL ASQUEZ_FACTORES.ASOCIADOS.AL.NIVEL_DATOS.PDF

23. Gomes-Leite-Morais, Roberta, Serra-Tanan, Tanan, da-Silva-Oliveira, Juliana, Pimentel-Macedo, Maiara, Alves-Nery, Adriana, Arcanjo-Matos-Filho, Silvo. Conocimientos y conductas de bioseguridad entre docentes de enfermería. Cuidado é Fundamental revista online de pesquisa. [revista en Internet] 2017 Enero. [citado 2017 Sep. 24]. 9(1): 137-143. Disponible http://www.seer.unirio.br/index.php/cuidadofundamental/article/view/5191/pdf

24. Moreno-Arroyo, M. Carmen, Puig-Llobet, Montserrat, Falcó-Pegueroles, Anna, Lluch-Canut, Ma. Teresa, Hidalgo-Blanco, Miguel, Miguel-Ruiz, Ma. Dolores, Roldán-Merino, Juan. La percepción del riesgo biológico en las prácticas clínico asistenciales en los estudiantes de Grado de Enfermería de la Universidad de Barcelona. Dipòsit Digital de la Universitat de Barcelona. [Tesis en Internet] 2015 Feb. [citado 2017 Sep 24]. Disponible en: http://diposit.ub.edu/dspace/handle/2445/95829 
25. Arrieta-Vergara, Katherine, Diaz-Cardenas, Shyrley, González-Martinez, Farith. Prevalencia de accidentes ocupacionales y factores relacionados en estudiantes de odontología. Rev. salud pública [revista en Internet]. 2013. [citado 2017 Sept 24]. 15 (1): 23-31. Disponible en: https://revistas.unal.edu.co/index.php/revsaludpublica/article/view/27303 